EUROPEAN JOURNAL OF PURE AND APPLIED MATHEMATICS

Vol. 12, No. 2, 2019, 605-621

ISSN 1307-5543 - www.ejpam.com

Published by New York Business Global

\title{
Combinatorial Identities with Generalized Higher-order Genocchi Sequences
}

\author{
Tian $\mathrm{Hao}^{1, *}$, Wuyungaowa ${ }^{1}$ \\ ${ }^{1}$ Department of Mathematical Sciences, \\ Inner Mongolia University, Hohhot, Inner Mongolia, P.R.China
}

\begin{abstract}
In this paper, we make use of the probabilistic method to calculate the moment representation of generalized higher-order Genocchi polynomials. We obtain the moment expression of the generalized higher-order Genocchi numbers with $a$ and $b$ parameters. Some characterizations and identities of generalized higher-order Genocchi polynomials are given by the proof of the moment expression. As far as properties given by predecessors are concerned, we prove them by the probabilistic method. Finally, new identities of relationships involving generalized higher-order Genocchi numbers and harmonic numbers, derangement numbers, Fibonacci numbers, Bell numbers, Bernoulli numbers, Euler numbers, Cauchy numbers and Stirling numbers of the second kind are established.
\end{abstract}

2010 Mathematics Subject Classifications: 11B68, 60E07, 11B83, 62E15.

Key Words and Phrases: Moment, Generating function, Generalized higher-order Genocchi numbers and polynomials, Laplace distribution.

\section{Introduction and Preliminaries}

The classical Genocchi numbers and polynomials play important roles in combinatorics. It is widely used in combinatorial mathematics, function theory, graph theory, approximate calculation and theoretical physics, such as the diffusion of matter. In the present paper, a further investigation for the generalized higher-order Genocchi polynomials with $a, b$ and $c$ parameters is performed. We depend on the Laplace distribution to calculate the moment representation of the generalized higher-order Genocchi polynomials. Hence the moment representation can also be used to give some relative identities and characterizations of the generalized higher-order Genocchi polynomials. Recently, symmetry identities of the generalized Bernoulli, Euler and Genocchi polynomials are investigated by Waseem A. Khan and other predecessors[6][7][8][9]. Here the probabilistic method provides great convenience to investigate symmetry identities of the generalized Genocchi polynomials

*Corresponding author.

DOI: https://doi.org/10.29020/nybg.ejpam.v12i2.3403

Email addresses: 1179827028@qq.com (T. Hao), wuyungw@163.com (Wuyungaowa) 
and relations between the generalized higher-order Genocchi numbers and combinatorial numbers.

Throughout this paper, let $t \in \mathbb{C}$ and $|t|<\pi /|\ln b-\ln a|, \alpha \in \mathbb{N}_{+}, x \in \mathbb{R}, a, b$ and $c$ are positive integers, $a \neq b$, we now turn to the generalized higher-order Genocchi polynomials $G_{n}^{(\alpha)}(x ; a, b, c)$ with $a, b$ and $c$ parameters for nonnegative integer n, which are usually defined by means of the following generating function[2][4, 5]:

$$
\left(\frac{2 t}{a^{t}+b^{t}}\right)^{\alpha} c^{x t}=\sum_{n=0}^{\infty} G_{n}^{(\alpha)}(x ; a, b, c) \frac{t^{n}}{n !} .
$$

Taking $x=0$ in Eq.(1), we get the generating function of the generalized higher-order Genocchi numbers $G_{n}^{(\alpha)}(a, b)$ with $a$ and $b$ parameters[4]:

$$
\left(\frac{2 t}{a^{t}+b^{t}}\right)^{\alpha}=\sum_{n=0}^{\infty} G_{n}^{(\alpha)}(a, b) \frac{t^{n}}{n !} .
$$

Setting $a=1, b=e, c=e$ in Eq.(1), the generating function of the classical higherorder Genocchi polynomials is as follows[3][5][9][12]:

$$
\left(\frac{2 t}{e^{t}+1}\right)^{\alpha} e^{x t}=\sum_{n=0}^{\infty} G_{n}^{(\alpha)}(x) \frac{t^{n}}{n !}
$$

then setting $x=0$ in Eq.(3), we get the generating function of the classical higher-order Genocchi numbers[1][12]:

$$
\left(\frac{2 t}{e^{t}+1}\right)^{\alpha}=\sum_{n=0}^{\infty} G_{n}^{(\alpha)} \frac{t^{n}}{n !} .
$$

In this paper, we make use of the special combinatorial sequences of the generalized Euler polynomials $E_{n}(x ; a, b, c)$ with $a, b$ and $c$, which are defined by the following generating function[10][11]:

$$
\frac{2 c^{x t}}{a^{t}+b^{t}}=\sum_{n=0}^{\infty} E_{n}(x ; a, b, c) \frac{t^{n}}{n !} .
$$

For $\alpha \in \mathbb{N}_{+}$, we get the generalized higher-order Euler polynomials $E_{n}^{(\alpha)}(x ; a, b, c)$ with $a, b$ and $c[11]$ :

$$
\left(\frac{2}{a^{t}+b^{t}}\right)^{\alpha} c^{x t}=\sum_{n=0}^{\infty} E_{n}^{(\alpha)}(x ; a, b, c) \frac{t^{n}}{n !} .
$$

We also need the following notations: r.v denotes a random variable, i.i.d shows that a sequence of random variables are independent and identically distributed. The notation $E$ denotes an expectation operator and definition is as follows: When $f(x)$ is a measurable function with continuous random variables $X$ and $p(x)$ is a density function of $X$, we have

$$
E f(X)=\int_{-\infty}^{+\infty} f(x) p(x) d x .
$$


Especially, setting $f(x)=x^{n}$, we obtain the moment of n-th order $E X^{n}$ of random variables.

Remark 1. [see 15] If $f$ and $g$ are exponential generating functions, and

$$
f g=\left(\sum_{r=0}^{\infty} \frac{a_{r} t^{r}}{r !}\right)\left(\sum_{s=0}^{\infty} \frac{b_{s} t^{s}}{s !}\right),
$$

then the coefficients of $\frac{t^{n}}{n !}$ in $f g$ are given by

$$
\left[\frac{t^{n}}{n !}\right](f g)=\sum_{r=0}^{n}\left(\begin{array}{l}
n \\
r
\end{array}\right) a_{r} b_{n-r} .
$$

Next we shall introduce several moment representations of some combinatorial sequences.

Lemma 1. [see 14] Suppose that r.v $u_{1}, u_{2}$, i.i.d $\sim U[0,1]$, then harmonic numbers $H_{n}=$ $\sum_{k=1}^{n} \frac{1}{k}$ have the following moment representation,

$$
H_{n}=n E\left(1-u_{1} u_{2}\right)^{n-1}, \quad n \geqslant 1 .
$$

Lemma 2. [see 14] Suppose that r.v $X \sim \Gamma(1,1)$, then derangement numbers $d_{n}=$ $n ! \sum_{k=0}^{n} \frac{(-1)^{k}}{k !}$ satisfy the following moment representation,

$$
d_{n}=E(X-1)^{n}, \quad n \geqslant 0 .
$$

Lemma 3. [see 14] Suppose that r.v $u \sim U[0,1]$, then Fibonacci numbers $F_{n}$ have the following moment representation,

$$
F_{n}=(n+1) E\left(\sqrt{5} u+\frac{(1-\sqrt{5})}{2}\right)^{n}, \quad n \geqslant 0 .
$$

Lemma 4. [see 14] Suppose that r.v $X \sim P(1)$, then Bell numbers $b_{n}$ have the following moment representation,

$$
\begin{aligned}
& b_{n}=E(X)^{n}, \quad n \geqslant 0, \\
& b_{n}=E(X+1)^{n-1}, \quad n \geqslant 1 .
\end{aligned}
$$

Lemma 5. [see 13] Suppose that r.v $L_{1}, L_{2}, \cdots$, i.i.d $\sim L[0,1]$ and r.v $L_{e}=\sum_{k \geq 1} \frac{L_{k}}{2 k \pi}$, then Bernoulli numbers $B_{n}$ satisfy the following moment representation,

$$
B_{n}=E\left(i L_{e}-\frac{1}{2}\right)^{n}, \quad n \geqslant 0 .
$$

Lemma 6. [see 13] Suppose that r.v $L_{1}, L_{2}, \cdots$, i.i.d $\sim L[0,1]$ and r.v $L=\sum_{k \geq 1} \frac{L_{k}}{(2 k-1) \pi}$, then Euler numbers $E_{n}$ have the following moment representation,

$$
E_{n}=2^{n} E(i L)^{n}, \quad n \geqslant 0 .
$$


Lemma 7. [see 14] Suppose that r. $v \sim \Gamma(u, 1), u \sim U[0,1]$, and $X, u$ are independent, then Cauchy numbers of the second kind $c_{n}$ satisfy the following moment representation,

$$
c_{n}=E X^{n}, \quad n \geqslant 0 .
$$

Lemma 8. [see 14] Suppose that r.v $u_{1}, u_{2}, \cdots$, i.i.d $\sim U[0,1]$ for all $i$, when $n, k \geqslant 1$, then Stirling numbers of the second kind have the following moment representation,

$$
S(n, k)=\left(\begin{array}{l}
n \\
k
\end{array}\right) E\left(u_{1}+u_{2}+\cdots+u_{k}\right)^{n-k} .
$$

It is demanded that $S(n, 0)=S(0, k)=0, S(0,0)=1$.

\section{Moment Representations of the Generalized Higher-order Genocchi polynomials}

In this section, we derive the moment representation of the generalized higher-order Genocchi polynomials by means of the probabilistic method. Some properties and identities of generalized higher-order Genocchi polynomials and numbers are given on the foundation of the moment expression.

Theorem 1. Suppose that r.v $L_{1}, L_{2}, \cdots$, i.i.d $\sim L[0,1], i^{2}=-1$, let $L=\sum_{k \geq 1} \frac{L_{k}}{(2 k-1) \pi}$ and for all $j, L^{(j)}=\sum_{k \geq 1} \frac{L_{k}^{(j)}}{(2 k-1) \pi}$ be random variables and r.v $\left\{L^{(j)}\right\}_{1 \leq j \leq \alpha}$ are independent and obey the same distribution as $L$. Then let $a, b$ and $c$ be positive integers with conditions $a \neq b, a b \neq 1$ and $c \neq 1$, for $\alpha \in \mathbb{N}_{+}, x \in \mathbb{R}, n \geq \alpha$, the generalized higher-order Genocchi polynomials $G_{n}^{(\alpha)}(x ; a, b, c)$ satisfy the following moment representation:

$$
G_{n}^{(\alpha)}(x ; a, b, c)=\frac{n !}{(n-\alpha) !} E\left[i\left(L^{(1)}+\cdots+L^{(\alpha)}\right) \ln \frac{b}{a}+x \ln c-\frac{\alpha}{2} \ln a b\right]^{n-\alpha}, \quad(n \geq \alpha) .
$$

Proof. From the generating function of the generalized higher-order Genocchi polynomials we discover

$$
\begin{aligned}
\sum_{n=0}^{\infty} G_{n}^{(\alpha)}(x ; a, b, c) \frac{t^{n}}{n !} & =\left(\frac{2 t}{b^{t}+a^{t}}\right)^{\alpha} c^{x t}=t^{\alpha}\left(\frac{2}{\left(\frac{b}{a}\right)^{t}+1}\right)^{\alpha} a^{-t \alpha} c^{x t} \\
& =t^{\alpha}\left(\frac{2 e^{\frac{t}{2} \ln \frac{b}{a}}}{e^{t \ln \frac{b}{a}}+1}\right)^{\alpha} e^{t\left(x \ln c-\frac{\alpha}{2} \ln \frac{b}{a}-\alpha \ln a\right)} \\
& =t^{\alpha}\left(\frac{2 e^{\frac{t}{2} \ln \frac{b}{a}}}{e^{t \ln \frac{b}{a}}+1}\right)^{\alpha} e^{t\left(x \ln c-\frac{\alpha}{2} \ln a b\right)} .
\end{aligned}
$$

Suppose that r.v $L_{1}, L_{2}, \cdots$, i.i.d $\sim L[0,1], i^{2}=-1, L=\sum_{k \geq 1} \frac{L_{k}}{(2 k-1) \pi}[13]$,

$$
\frac{2 e^{\frac{t}{2}}}{e^{t}+1}=\frac{1}{\frac{e^{\frac{t}{2}}+e^{-\frac{t}{2}}}{2}}=\frac{1}{\cosh \left(\frac{t}{2}\right)}=\prod_{k \geq 1}\left[1+\left(\frac{t}{(2 k-1) \pi}\right)^{2}\right]^{-1} .
$$


Because $\left[1+\left(\frac{t}{(2 k-1) \pi}\right)^{2}\right]^{-1}$ is the characteristic function of r.v $\frac{L_{k}}{(2 k-1) \pi}$, we discover that $\prod_{k \geq 1}\left[1+\left(\frac{t}{(2 k-1) \pi}\right)^{2}\right]^{-1}$ is the characteristic function of $L=\sum_{k \geq 1} \frac{L_{k}}{(2 k-1) \pi}$. All moments of the random variables exist, hence the discovery depends on the following identity:

$$
\sum_{n=0}^{\infty} E L^{n} \frac{(i t)^{n}}{n !}=\prod_{k \geq 1}\left[1+\left(\frac{t}{(2 k-1) \pi}\right)^{2}\right]^{-1}=\frac{2 e^{\frac{t}{2}}}{e^{t}+1}, \quad\left(i^{2}=-1\right) .
$$

Let $L^{(j)}=\sum_{k \geq 1} \frac{L_{k}^{(j)}}{(2 k-1) \pi}$ be a random variable and for all $j$ r.v $\left\{L^{(j)}\right\}_{1 \leq j \leq \alpha}$ are independent and obey the same distribution as L. According to Eq.(16) and Eq.(18), we have

$$
\begin{aligned}
t^{\alpha} & \left(\sum_{m \geq 0} E L^{m} \frac{\left(i t \ln \frac{b}{a}\right)^{m}}{m !}\right)^{\alpha} e^{t\left(x \ln c-\frac{\alpha}{2} \operatorname{lnab}\right)} \\
= & t^{\alpha}\left\{\sum_{n \geq 0} \sum_{m_{1}+\cdots+m_{\alpha}=n}\left(\begin{array}{c}
n \\
m_{1}, \cdots, m_{\alpha}
\end{array}\right) E\left(i L^{(1)} \ln \frac{b}{a}\right)^{m_{1}} \cdots E\left(i L^{(\alpha)} \ln \frac{b}{a}\right)^{m_{\alpha}} \frac{t^{n}}{n !}\right\} \\
& \times\left\{\sum_{n \geq 0}\left(x \ln c-\frac{\alpha}{2} \ln a b\right)^{n} \frac{t^{n}}{n !}\right\} \\
= & t^{\alpha} \sum_{n \geq 0} E\left(i L^{(1)} \ln \frac{b}{a}+\cdots+i L^{(\alpha)} \ln \frac{b}{a}\right)^{n} \frac{t^{n}}{n !} \frac{\sum}{n \geq 0}\left(x \ln c-\frac{\alpha}{2} \ln a b\right)^{n} \frac{t^{n}}{n !} \\
= & \sum_{n \geq 0} \sum_{k=0}^{n}\left(\begin{array}{l}
n \\
k
\end{array}\right) E\left[i\left(L^{(1)}+\cdots+L^{(\alpha)}\right) \ln \frac{b}{a}\right]^{k}\left(x \ln c-\frac{\alpha}{2} \ln a b\right)^{n-k} \frac{t^{n+\alpha}}{n !} \\
= & \sum_{n \geq 0} E\left[i\left(L^{(1)}+\cdots+L^{(\alpha)}\right) \ln \frac{b}{a}+x \ln c-\frac{\alpha}{2} \ln a b\right]^{n} \frac{t^{n+\alpha}}{n !} \\
= & \sum_{n \geq \alpha} \frac{n !}{(n-\alpha) !} E\left[i\left(L^{(1)}+\cdots+L^{(\alpha)}\right) \ln \frac{b}{a}+x \ln c-\frac{\alpha}{2} \ln a b\right]^{n-\alpha} \frac{t^{n}}{n !}
\end{aligned}
$$

By comparing the coefficients $\frac{t^{n}}{n !}$, we arrive at the moment expression of $G_{n}^{(\alpha)}(x ; a, b, c)$.

Corollary 1. Taking $x=0$ in Eq.(15), we get the moment representation of the higherorder Genocchi numbers with $a$ and $b$ parameters $G_{n}^{(\alpha)}(a, b)$,

$$
G_{n}^{(\alpha)}(0 ; a, b)=G_{n}^{(\alpha)}(a, b)=\frac{n !}{(n-\alpha) !} E\left[i\left(L^{(1)}+\cdots+L^{(\alpha)}\right) \ln \frac{b}{a}-\frac{\alpha}{2} \ln a b\right]^{n-\alpha},(n \geq \alpha) .
$$

For $a=1, b=e$ in Eq.(19), we get the moment representation of the higher-order Genocchi numbers $G_{n}^{(\alpha)}$,

$$
G_{n}^{(\alpha)}(1, e)=G_{n}^{(\alpha)}=\frac{n !}{(n-\alpha) !} E\left(i L^{(1)}+\cdots+i L^{(\alpha)}-\frac{\alpha}{2}\right)^{n-\alpha}, \quad(n \geq \alpha) .
$$


Corollary 2. For $\alpha=1$ in Eq.(15), we get the moment representation of the generalized Genocchi polynomials $G_{n}(x ; a, b, c)$,

$$
G_{n}^{(1)}(x ; a, b, c)=G_{n}(x ; a, b, c)=n E\left(i \operatorname{Lln} \frac{b}{a}+x \ln c-\frac{1}{2} \ln a b\right)^{n-1}, \quad(n \geq 1) .
$$

Corollary 3. Setting $a=1, b=e, c=e$ in Eq.(15), we get the moment representation of the higher-order Genocchi polynomials $G_{n}^{(\alpha)}(x)$,

$$
G_{n}^{(\alpha)}(x ; 1, e, e)=G_{n}^{(\alpha)}(x)=\frac{n !}{(n-\alpha) !} E\left[i L^{(1)}+\cdots+i L^{(\alpha)}+x-\frac{\alpha}{2}\right]^{n-\alpha}, \quad(n \geq \alpha) .
$$

Corollary 4. We can easily obtain the moment representation of the generalized higherorder Euler polynomials and get the relation between generalized higher-order Euler polynomials and generalized higher-order Gennocchi polynomials in the proof of theorem 1,

$$
\begin{gathered}
E_{n}^{(\alpha)}(x ; a, b, c)=E\left[i\left(L^{(1)}+\cdots+L^{(\alpha)}\right) \ln \frac{b}{a}+x \ln c-\frac{\alpha}{2} \ln a b\right]^{n}, \\
G_{n}^{(\alpha)}(x ; a, b, c)=\frac{n !}{(n-\alpha) !} E_{n-\alpha}^{(\alpha)}(x ; a, b, c), \quad(n \geq \alpha) .
\end{gathered}
$$

The generalized higher-order Euler polynomials and the generalized higher-order Genocchi polynomials have similar properties and forms, we only aim at the properties of generalized higher-order Genocchi polynomials here.

Theorem 2. Let $a, b$ and $c$ be positive integers with conditions $a \neq b, a b \neq 1$ and $c \neq 1$, for $\alpha \in \mathbb{N}_{+}, x \in \mathbb{R}, n \geq \alpha$, then we get

$$
G_{n}^{(\alpha)}(x+\alpha ; a, b, c)=G_{n}^{(\alpha)}\left(x ; \frac{a}{c}, \frac{b}{c}, c\right) .
$$

Proof. In light of the theorem 1 we have

$$
\begin{aligned}
& G_{n}^{(\alpha)}(x+\alpha ; a, b, c)=\frac{n !}{(n-\alpha) !} E\left[i\left(L^{(1)}+\cdots+L^{(\alpha)}\right) \ln \frac{b}{a}+(x+\alpha) \ln c-\frac{\alpha}{2} \ln a b\right]^{n-\alpha} \\
& =\frac{n !}{(n-\alpha) !} E\left[i\left(L^{(1)}+\cdots+L^{(\alpha)}\right) \ln \frac{b}{a}+x \ln c+\frac{\alpha}{2} \ln c^{2}-\frac{\alpha}{2} \ln a b\right]^{n-\alpha} \\
& =\frac{n !}{(n-\alpha) !} E\left[i\left(L^{(1)}+\cdots+L^{(\alpha)}\right) \ln \frac{\frac{b}{\frac{a}{a}}}{\frac{a}{c}}+x \ln c-\frac{\alpha}{2} \ln \frac{a}{c} \frac{b}{c}\right]^{n-\alpha}=G_{n}^{(\alpha)}\left(x ; \frac{a}{c}, \frac{b}{c}, c\right) .
\end{aligned}
$$

This concludes the proof.

Theorem 3. Let $a, b$ and $c$ be positive integers with conditions $a \neq b, a b \neq 1$ and $c \neq 1$, for $\alpha \in \mathbb{N}_{+}, x \in \mathbb{R}, n \geq \alpha$, then we get

$$
G_{n}^{(\alpha)}(\alpha-x ; a, b, c)=(-1)^{n-\alpha} G_{n}^{(\alpha)}\left(x ; \frac{c}{a}, \frac{c}{b}, c\right)=G_{n}^{(\alpha)}\left(-x ; \frac{a}{c}, \frac{b}{c}, c\right) .
$$


Proof. According to theorem 1, we have

$$
\begin{aligned}
& G_{n}^{(\alpha)}(\alpha-x ; a, b, c)=\frac{n !}{(n-\alpha) !} E\left[i\left(L^{(1)}+\cdots+L^{(\alpha)}\right) \ln \frac{b}{a}+(\alpha-x) \ln c-\frac{\alpha}{2} \ln a b\right]^{n-\alpha} \\
& =\frac{n !}{(n-\alpha) !}(-1)^{n-\alpha} E\left[i\left(L^{(1)}+\cdots+L^{(\alpha)}\right) \ln \frac{\frac{1}{b}}{\frac{1}{a}}+x \ln c-\frac{\alpha}{2} \ln c^{2}-\frac{\alpha}{2} \ln \frac{1}{a b}\right]^{n-\alpha} \\
& =\frac{n !}{(n-\alpha) !}(-1)^{n-\alpha} E\left[i\left(L^{(1)}+\cdots+L^{(\alpha)}\right) \ln \frac{\frac{c}{b}}{\frac{c}{a}}+x \ln c-\frac{\alpha}{2} \ln \frac{c}{a} \frac{c}{b}\right]^{n-\alpha} \\
& =(-1)^{n-\alpha} G_{n}^{(\alpha)}\left(x ; \frac{c}{a}, \frac{c}{b}, c\right) .
\end{aligned}
$$

On substituting $\mathrm{x}$ with $-\mathrm{x}$ in Eq.(25), then Eq.(26) arises.

Theorem 4. Let $a, b$ and $c$ be positive integers with conditions $a \neq b, a b \neq 1$ and $c \neq 1$, for $\alpha \in \mathbb{N}_{+}, x \in \mathbb{R}, n \geq \alpha$, then we get

$$
G_{n}^{(\alpha)}(-x ; a, b, c)=G_{n}^{(\alpha)}\left(x ; a, b, \frac{1}{c}\right) .
$$

Proof. With the help of theorem 1 we have

$$
\begin{aligned}
& G_{n}^{(\alpha)}(-x ; a, b, c)=\frac{n !}{(n-\alpha) !} E\left[i\left(L^{(1)}+\cdots+L^{(\alpha)}\right) \ln \frac{b}{a}-x \ln c-\frac{\alpha}{2} \ln a b\right]^{n-\alpha} \\
& =\frac{n !}{(n-\alpha) !} E\left[i\left(L^{(1)}+\cdots+L^{(\alpha)}\right) \ln \frac{b}{a}+x \ln \frac{1}{c}-\frac{\alpha}{2} \ln a b\right]^{n-\alpha}=G_{n}^{(\alpha)}\left(x ; a, b, \frac{1}{c}\right) .
\end{aligned}
$$

This concludes the proof.

Corollary 5. Taking $\alpha=1$ in Eq.(25), Eq.(26), Eq.(27), we can easily get the following identities,

$$
\begin{gathered}
G_{n}(x+1 ; a, b, c)=G_{n}\left(x ; \frac{a}{c}, \frac{b}{c}, c\right), \\
G_{n}(1-x ; a, b, c)=(-1)^{n-1} G_{n}\left(x ; \frac{c}{a}, \frac{c}{b}, c\right)=G_{n}\left(-x ; \frac{a}{c}, \frac{b}{c}, c\right), \\
G_{n}(-x ; a, b, c)=G_{n}\left(x ; a, b, \frac{1}{c}\right) .
\end{gathered}
$$

Theorem 5. Let $a, b$ and $c$ be positive integers with conditions $a \neq b, a b \neq 1$ and $c \neq 1$, for $\alpha \in \mathbb{N}_{+}, x \in \mathbb{R}, n \geq \alpha$, then we get

$$
G_{n}^{(\alpha)}(x ; a, b, c)=\left(\ln \frac{b}{a}\right)^{n-\alpha} G_{n}^{(\alpha)}\left(\frac{x \ln c-\alpha \ln a}{\ln b-\ln a}\right) .
$$


Proof. From theorem 1 we have

$$
\begin{aligned}
& G_{n}^{(\alpha)}(x ; a, b, c)=\frac{n !}{(n-\alpha) !} E\left[i\left(L^{(1)}+\cdots+L^{(\alpha)}\right) \ln \frac{b}{a}+x \ln c-\frac{\alpha}{2} \ln a b\right]^{n-\alpha} \\
& =\left(\ln \frac{b}{a}\right)^{n-\alpha} \frac{n !}{(n-\alpha) !} E\left[i\left(L^{(1)}+\cdots+L^{(\alpha)}\right)+\frac{x \ln c}{\ln b-\ln a}-\frac{\frac{\alpha}{2} \ln b+\frac{\alpha}{2} \ln a}{\ln b-\ln a}\right]^{n-\alpha} \\
& =\left(\ln \frac{b}{a}\right)^{n-\alpha} \frac{n !}{(n-\alpha) !} E\left[i\left(L^{(1)}+\cdots+L^{(\alpha)}\right)+\frac{x \ln c}{\ln b-\ln a}-\frac{\frac{\alpha}{2} \ln b-\frac{\alpha}{2} \ln a+\alpha \ln a}{\ln b-\ln a}\right]^{n-\alpha} \\
& =\left(\ln \frac{b}{a}\right)^{n-\alpha} \frac{n !}{(n-\alpha) !} E\left[i\left(L^{(1)}+\cdots+L^{(\alpha)}\right)+\frac{x \ln c-\alpha \ln a}{\ln b-\ln a}-\frac{\alpha}{2}\right]^{n-\alpha} \\
& =\left(\ln \frac{b}{a}\right)^{n-\alpha} G_{n}^{(\alpha)}\left(\frac{x \ln c-\alpha \ln a}{\ln b-\ln a}\right) .
\end{aligned}
$$

Thus we arrive at the desired result.

Theorem 6. Let $a, b$ and $c$ be positive integers with conditions $a \neq b, a b \neq 1$ and $c \neq 1$, for $\alpha \in \mathbb{N}_{+}, x \in \mathbb{R}, n \geq \alpha$, then we get

$$
(-1)^{n-\alpha} G_{n}^{(\alpha)}(x ; a, b, c)=\sum_{k=\alpha}^{n}\left(\begin{array}{l}
n \\
k
\end{array}\right) G_{k}^{(\alpha)}(-x ; b, a, c)(\alpha \ln a b)^{n-k} .
$$

Proof. From Eq.(15) in theorem 1, we have

$$
\begin{aligned}
& (-1)^{n-\alpha} G_{n}^{(\alpha)}(x ; a, b, c)=\frac{n !}{(n-\alpha) !} E\left[i\left(L^{(1)}+\cdots+L^{(\alpha)}\right) \ln \frac{a}{b}-x \ln c-\frac{\alpha}{2} \ln \frac{1}{a b}\right]^{n-\alpha} \\
& =\frac{n !}{(n-\alpha) !} E\left[i\left(L^{(1)}+\cdots+L^{(\alpha)}\right) \ln \frac{a}{b}-x \ln c-\frac{\alpha}{2} \ln \frac{1}{a b}-\frac{\alpha}{2} \ln (a b)^{2}+\frac{\alpha}{2} \ln (a b)^{2}\right]^{n-\alpha} \\
& =\frac{n !}{(n-\alpha) !} E\left[i\left(L^{(1)}+\cdots+L^{(\alpha)}\right) \ln \frac{a}{b}-x \ln c-\frac{\alpha}{2} \ln a b+\alpha \ln a b\right]^{n-\alpha} \\
& =\frac{n !}{(n-\alpha) !} \sum_{k=\alpha}^{n}\left(\begin{array}{l}
n-\alpha \\
k-\alpha
\end{array}\right) E\left[i\left(L^{(1)}+\cdots+L^{(\alpha)}\right) \ln \frac{a}{b}-x \ln c-\frac{\alpha}{2} \ln a b\right]^{k-\alpha}(\alpha \ln a b)^{n-k} \\
& =\sum_{k=\alpha}^{n}\left(\begin{array}{l}
n \\
k
\end{array}\right) G_{k}^{(\alpha)}(-x ; b, a, c)(\alpha \ln a b)^{n-k} .
\end{aligned}
$$

Therefor we derive the Eq.(32).

Theorem 7. Let $a, b$ and $c$ be positive integers with conditions $a \neq b, a b \neq 1$ and $c \neq 1$, for $\alpha \in \mathbb{N}_{+}, x \in \mathbb{R}, n \geq \alpha$, then we get

$$
\sum_{k=\alpha}^{n}\left(\begin{array}{l}
n \\
k
\end{array}\right) G_{k}^{(\alpha)}(a, b) x^{n-k}(\ln c)^{n-k}=\sum_{k=\alpha}^{n}\left(\begin{array}{l}
n \\
k
\end{array}\right)(-\alpha \ln c)^{n-k} G_{k}^{(\alpha)}\left(x ; \frac{a}{c}, \frac{b}{c}, c\right) .
$$


Proof. By theorem 1 we have

$$
\begin{aligned}
& G_{n}^{(\alpha)}(x ; a, b, c)=\frac{n !}{(n-\alpha) !} E\left[i\left(L^{(1)}+\cdots+L^{(\alpha)}\right) \ln \frac{b}{a}+x \ln c-\frac{\alpha}{2} \ln a b\right]^{n-\alpha} \\
& =\frac{n !}{(n-\alpha) !} \sum_{k=\alpha}^{n}\left(\begin{array}{c}
n-\alpha \\
k-\alpha
\end{array}\right) E\left[i\left(L^{(1)}+\cdots+L^{(\alpha)}\right) \ln \frac{b}{a}-\frac{\alpha}{2} \ln a b\right]^{k-\alpha}(x \ln c)^{n-k} \\
& =\sum_{k=\alpha}^{n}\left(\begin{array}{l}
n \\
k
\end{array}\right) G_{k}^{(\alpha)}(a, b) x^{n-k}(\ln c)^{n-k},
\end{aligned}
$$

and

$$
\begin{aligned}
& G_{n}^{(\alpha)}(x ; a, b, c)=\frac{n !}{(n-\alpha) !} E\left[i\left(L^{(1)}+\cdots+L^{(\alpha)}\right) \ln \frac{b}{a}+x \ln c-\frac{\alpha}{2} \ln a b\right]^{n-\alpha} \\
& =\frac{n !}{(n-\alpha) !} \sum_{k=\alpha}^{n}\left(\begin{array}{l}
n-\alpha \\
k-\alpha
\end{array}\right) E\left[i\left(L^{(1)}+\cdots+L^{(\alpha)}\right) \ln \frac{\frac{b}{a}}{\frac{a}{c}}+x \ln c-\frac{\alpha}{2} \ln \frac{a b}{c^{2}}\right]^{k-\alpha}(-\alpha \ln c)^{n-k} \\
& =\sum_{k=\alpha}^{n}\left(\begin{array}{l}
n \\
k
\end{array}\right)(-\alpha \ln c)^{n-k} G_{k}^{(\alpha)}\left(x ; \frac{a}{c}, \frac{b}{c}, c\right) .
\end{aligned}
$$

Thus, combining Eq.(34) and Eq.(35) gives the theorem 7.

Theorem 8. Let $a, b$ and $c$ be positive integers with conditions $a \neq b, a b \neq 1$ and $c \neq 1$, for $\alpha \in \mathbb{N}_{+}, x, y \in \mathbb{R}, n \geq \alpha$, then we get

$$
G_{n}^{(\alpha)}(x+y ; a, b, c)=\sum_{k=\alpha}^{n}\left(\begin{array}{l}
n \\
k
\end{array}\right) G_{k}^{(\alpha)}(x ; a, b, c) y^{n-k}(\ln c)^{n-k} .
$$

Proof. It follows from Eq.(15) that

$$
\begin{aligned}
& G_{n}^{(\alpha)}(x+y ; a, b, c)=\frac{n !}{(n-\alpha) !} E\left[i\left(L^{(1)}+\cdots+L^{(\alpha)}\right) \ln \frac{b}{a}+(x+y) \ln c-\frac{\alpha}{2} \ln a b\right]^{n-\alpha} \\
& =\frac{n !}{(n-\alpha) !} \sum_{k=\alpha}^{n}\left(\begin{array}{l}
n-\alpha \\
k-\alpha
\end{array}\right) E\left[i\left(L^{(1)}+\cdots+L^{(\alpha)}\right) \ln \frac{b}{a}+x \ln c-\frac{\alpha}{2} \ln a b\right]^{k-\alpha}(y \ln c)^{n-k} \\
& =\frac{n !}{(n-\alpha) !} \sum_{k=\alpha}^{n}\left(\begin{array}{l}
n-\alpha \\
k-\alpha
\end{array}\right) \frac{(k-\alpha) !}{k !} G_{k}^{(\alpha)}(x ; a, b, c)(y \ln c)^{n-k} \\
& =\sum_{k=\alpha}^{n}\left(\begin{array}{l}
n \\
k
\end{array}\right) G_{k}^{(\alpha)}(x ; a, b, c) y^{n-k}(\ln c)^{n-k} .
\end{aligned}
$$

This concludes the proof. 
Theorem 9. Let $a, b$ and $c$ be positive integers with conditions $a \neq b, a b \neq 1$ and $c \neq 1$, for $\alpha \in \mathbb{N}_{+}, x, y, z \in \mathbb{R}, n \geq \alpha$, then we get

$$
\begin{gathered}
\int_{x}^{y} G_{n}^{(\alpha)}(z ; a, b, c) d z=\frac{1}{(n+1) \operatorname{lnc}}\left[G_{n+1}^{(\alpha)}(y ; a, b, c)-G_{n+1}^{(\alpha)}(x ; a, b, c)\right], \\
\frac{\partial^{l} G_{n}^{(\alpha)}(x ; a, b, c)}{\partial x^{l}}=(n)_{l}(\operatorname{lnc})^{l} G_{n-l}^{(\alpha)}(x ; a, b, c) .
\end{gathered}
$$

Proof. By Eq.(15), we have

$$
\begin{aligned}
& \int_{x}^{y} G_{n}^{(\alpha)}(z ; a, b, c) d z=\int_{x}^{y} \frac{n !}{(n-\alpha) !} E\left[i\left(L^{(1)}+\cdots+L^{(\alpha)}\right) \ln \frac{b}{a}+z \ln c-\frac{\alpha}{2} \ln a b\right]^{n-\alpha} d z \\
& =\frac{n !}{(n-\alpha) !} E \int_{x}^{y}\left[i\left(L^{(1)}+\cdots+L^{(\alpha)}\right) \ln \frac{b}{a}+z \ln c-\frac{\alpha}{2} \ln a b\right]^{n-\alpha} d z \\
& =\frac{n !}{(n-\alpha) !} \frac{1}{(n-\alpha+1) \ln c}\left\{E\left[i\left(L^{(1)}+\cdots+L^{(\alpha)}\right) \ln \frac{b}{a}+y \ln c-\frac{\alpha}{2} \ln a b\right]^{n-\alpha+1}\right. \\
& \left.\quad-E\left[i\left(L^{(1)}+\cdots+L^{(\alpha)}\right) \ln \frac{b}{a}+x \ln c-\frac{\alpha}{2} \ln a b\right]^{n-\alpha+1}\right\} \\
& =\frac{n !}{(n-\alpha) !} \frac{1}{(n-\alpha+1) \ln c} \frac{(n-\alpha+1) !}{(n+1) !}\left[G_{n+1}^{(\alpha)}(y ; a, b, c)-G_{n+1}^{(\alpha)}(x ; a, b, c)\right] \\
& =\frac{1}{(n+1) \ln c}\left[G_{n+1}^{(\alpha)}(y ; a, b, c)-G_{n+1}^{(\alpha)}(x ; a, b, c)\right] .
\end{aligned}
$$

As for Eq.(38), we have

$$
\begin{aligned}
& \frac{\partial^{l} G_{n}^{(\alpha)}(x ; a, b, c)}{\partial x^{l}}=\frac{\partial^{l}\left\{\frac{n !}{(n-\alpha) !} E\left[i\left(L^{(1)}+\cdots+L^{(\alpha)}\right) \ln \frac{b}{a}+x \ln c-\frac{\alpha}{2} \ln a b\right]^{n-\alpha}\right\}}{\partial x^{l}} \\
& =\frac{n !}{(n-\alpha) !}(\ln c)^{l}(n-\alpha)(n-\alpha-1) \cdots(n-\alpha-l+1) \\
& \quad \times E\left[i\left(L^{(1)}+\cdots+L^{(\alpha)}\right) \ln \frac{b}{a}+x \ln c-\frac{\alpha}{2} \ln a b\right]^{n-\alpha-l} \\
& =\frac{n !(\ln c)^{l}}{(n-\alpha) !} \frac{(n-\alpha) !}{(n-\alpha-l) !} \frac{(n-\alpha-l) !}{(n-l) !} G_{n-l}^{(\alpha)}(x ; a, b, c) \\
& =(n)_{l}(\operatorname{lnc})^{l} G_{n-l}^{(\alpha)}(x ; a, b, c) .
\end{aligned}
$$

This concludes the proof.

Theorem 10. Let $a, b$ and $c$ be positive integers with conditions $a \neq b, a b \neq 1$ and $c \neq 1$, for $\alpha \in \mathbb{N}_{+}, x \in \mathbb{R}, n \geq \alpha$, for all $d, l \in \mathbb{N}$, then we get

$$
\begin{aligned}
& \sum_{k=\alpha}^{n-\alpha} \sum_{j=\alpha}^{k}\left(\begin{array}{l}
n \\
k
\end{array}\right)\left(\begin{array}{l}
k \\
j
\end{array}\right) d^{n-k} l^{k}(-\alpha l n c)^{k-j} G_{n-k}^{(\alpha)}(l x ; a, b, c) G_{j}^{(\alpha)}\left(d x ; \frac{a}{c}, \frac{b}{c}, c\right) \\
& =\sum_{k=\alpha}^{n-\alpha} \sum_{j=\alpha}^{k}\left(\begin{array}{l}
n \\
k
\end{array}\right)\left(\begin{array}{l}
k \\
j
\end{array}\right) l^{n-k} d^{k}(-\alpha \ln c)^{k-j} G_{n-k}^{(\alpha)}(d x ; a, b, c) G_{j}^{(\alpha)}\left(l x ; \frac{a}{c}, \frac{b}{c}, c\right) .
\end{aligned}
$$


Proof. Firstly, we have

$$
\begin{aligned}
& \frac{n !}{(n-2 \alpha) !} E\left[i(d+l)\left(L^{(1)}+\cdots+L^{(\alpha)}\right) \ln \frac{b}{a}+2 d l x \ln c-\frac{\alpha}{2}(d+l) \ln a b\right]^{n-2 \alpha} \\
& =\frac{n !}{(n-2 \alpha) !} \sum_{k=\alpha}^{n-2 \alpha}\left(\begin{array}{c}
n-2 \alpha \\
k-\alpha
\end{array}\right) E\left[i d\left(L^{(1)}+\cdots+L^{(\alpha)}\right) \ln \frac{b}{a}+d l x \ln c-\frac{\alpha d}{2} \ln a b\right]^{n-k-\alpha} \\
& \quad \times E\left[i l\left(L^{(1)}+\cdots+L^{(\alpha)}\right) \ln \frac{b}{a}+d l x \ln c-\frac{\alpha l}{2} \ln a b\right]^{k-\alpha} \\
& =\frac{(d l)^{-\alpha} n !}{(n-2 \alpha) !} \sum_{k=\alpha}^{n-\alpha}\left(\begin{array}{c}
n-2 \alpha \\
k-\alpha
\end{array}\right) \frac{(n-k-\alpha) ! d^{n-k} G_{n-k}^{(\alpha)}(l x ; a, b, c)}{(n-k) !} \frac{(k-\alpha) ! l^{k} G_{k}^{(\alpha)}(d x ; a, b, c)}{k !} \\
& =(d l)^{-\alpha} \sum_{k=\alpha}^{n-\alpha}\left(\begin{array}{l}
n \\
k
\end{array}\right) d^{n-k} l^{k} G_{n-k}^{(\alpha)}(l x ; a, b, c) G_{k}^{(\alpha)}(d x ; a, b, c),
\end{aligned}
$$

according to Eq.(35),

$$
\begin{aligned}
& (d l)^{-\alpha} \sum_{k=\alpha}^{n-\alpha}\left(\begin{array}{l}
n \\
k
\end{array}\right) d^{n-k} l^{k} G_{n-k}^{(\alpha)}(l x ; a, b, c) G_{k}^{(\alpha)}(d x ; a, b, c) \\
& =(d l)^{-\alpha} \sum_{k=\alpha}^{n-\alpha}\left(\begin{array}{l}
n \\
k
\end{array}\right) d^{n-k} l^{k} G_{n-k}^{(\alpha)}(l x ; a, b, c) \sum_{j=\alpha}^{k}\left(\begin{array}{l}
k \\
j
\end{array}\right)(-\alpha l n c)^{k-j} G_{j}^{(\alpha)}\left(d x ; \frac{a}{c}, \frac{b}{c}, c\right) .
\end{aligned}
$$

Secondly, we have

$$
\begin{aligned}
& \frac{n !}{(n-2 \alpha) !} E\left[i(d+l)\left(L^{(1)}+\cdots+L^{(\alpha)}\right) \ln \frac{b}{a}+2 d l x \ln c-\frac{\alpha}{2}(d+l) \ln a b\right]^{n-2 \alpha} \\
& =\frac{n !}{(n-2 \alpha) !} \sum_{k=\alpha}^{n-2 \alpha}\left(\begin{array}{c}
n-2 \alpha \\
k-\alpha
\end{array}\right) E\left[i l\left(L^{(1)}+\cdots+L^{(\alpha)}\right) \ln \frac{b}{a}+d l x \ln c-\frac{\alpha l}{2} \ln a b\right]^{n-k-\alpha} \\
& \quad \times E\left[i d\left(L^{(1)}+\cdots+L^{(\alpha)}\right) \ln \frac{b}{a}+d l x \ln c-\frac{\alpha d}{2} \ln a b\right]^{k-\alpha} \\
& =\frac{(l d)^{-\alpha} n !}{(n-2 \alpha) !} \sum_{k=\alpha}^{n-\alpha}\left(\begin{array}{c}
n-2 \alpha \\
k-\alpha
\end{array}\right) \frac{(n-k-\alpha) ! l^{n-k} G_{n-k}^{(\alpha)}(d x ; a, b, c)}{(n-k) !} \frac{(k-\alpha) ! d^{k} G_{k}^{(\alpha)}(l x ; a, b, c)}{k !} \\
& =(l d)^{-\alpha} \sum_{k=\alpha}^{n-\alpha}\left(\begin{array}{l}
n \\
k
\end{array}\right) l^{n-k} d^{k} G_{n-k}^{(\alpha)}(d x ; a, b, c) G_{k}^{(\alpha)}(l x ; a, b, c) \\
& =(l d)^{-\alpha} \sum_{k=\alpha}^{n-\alpha}\left(\begin{array}{l}
n \\
k
\end{array}\right) l^{n-k} d^{k} G_{n-k}^{(\alpha)}(d x ; a, b, c) \sum_{j=\alpha}^{k}\left(\begin{array}{l}
k \\
j
\end{array}\right)(-\alpha l n c)^{k-j} G_{j}^{(\alpha)}\left(l x ; \frac{a}{c}, \frac{b}{c}, c\right) .
\end{aligned}
$$

By equating Eq.(40) and Eq.(41), we arrive at the desired result. 
From the proof of theorem 10, we get the following identity:

$$
\begin{aligned}
& \sum_{k=\alpha}^{n-\alpha}\left(\begin{array}{l}
n \\
k
\end{array}\right) d^{n-k} l^{k} G_{n-k}^{(\alpha)}(l x ; a, b, c) G_{k}^{(\alpha)}(d x ; a, b, c) \\
& =\sum_{k=\alpha}^{n-\alpha}\left(\begin{array}{l}
n \\
k
\end{array}\right) l^{n-k} d^{k} G_{n-k}^{(\alpha)}(d x ; a, b, c) G_{k}^{(\alpha)}(l x ; a, b, c) .
\end{aligned}
$$

Corollary 6. Setting $\alpha=1$ in Eq.(39), we obtain

$$
\begin{aligned}
& \sum_{k=1}^{n-1} \sum_{j=1}^{k}\left(\begin{array}{l}
n \\
k
\end{array}\right)\left(\begin{array}{l}
k \\
j
\end{array}\right) d^{n-k} l^{k}(-\ln c)^{k-j} G_{n-k}(l x ; a, b, c) G_{j}\left(d x ; \frac{a}{c}, \frac{b}{c}, c\right) \\
& =\sum_{k=1}^{n-1} \sum_{j=1}^{k}\left(\begin{array}{l}
n \\
k
\end{array}\right)\left(\begin{array}{l}
k \\
j
\end{array}\right) l^{n-k} d^{k}(-\ln c)^{k-j} G_{n-k}(d x ; a, b, c) G_{j}\left(l x ; \frac{a}{c}, \frac{b}{c}, c\right) .
\end{aligned}
$$

Then taking $a=1, b=e, c=e$ in Eq.(43), we get

$$
\sum_{k=1}^{n-1} \sum_{j=1}^{k}\left(\begin{array}{l}
n \\
k
\end{array}\right)\left(\begin{array}{l}
k \\
j
\end{array}\right) d^{n-k} l^{k} G_{n-k}(l x) G_{j}(d x)=\sum_{k=1}^{n-1} \sum_{j=1}^{k}\left(\begin{array}{l}
n \\
k
\end{array}\right)\left(\begin{array}{l}
k \\
j
\end{array}\right) l^{n-k} d^{k} G_{n-k}(d x) G_{j}(l x) .
$$

\section{Identities of Generalized Higher-order Genocchi Numbers and Special Combinatorial Sequences}

In this section, we establish some new identities involving the generalized higher-order Genocchi numbers and harmonic numbers, derangement numbers, Fibonacci numbers, Bell numbers, Bernoulli numbers, Euler numbers, Cauchy numbers and Stirling numbers of second kind by means of the moment representation of the generalized higher-order Genocchi polynomials.

Theorem 11. Let $a, b$ and $c$ be positive integers with conditions $a \neq b, a b \neq 1$ and $c \neq 1$, for $\alpha \in \mathbb{N}_{+}, x \in \mathbb{R}, n \geq \alpha$, then we get

$$
\begin{gathered}
G_{n}^{(\alpha)}(x ; a, b, c)=\sum_{k=\alpha}^{n}\left(\begin{array}{l}
n \\
k
\end{array}\right)(\ln c)^{n-k} x^{n-k} G_{k}^{(\alpha)}(a, b), \\
G_{n}^{(\alpha)}(x ; a, b, c)=\sum_{k=\alpha}^{n}\left(\begin{array}{l}
n \\
k
\end{array}\right)(\ln c)^{n-k}\left(\ln \frac{b}{a}\right)^{k-\alpha} x^{n-k} G_{k}^{(\alpha)}\left(\frac{\alpha \ln a}{\ln a-\ln b}\right), \\
G_{n}^{(\alpha)}(x ; a, b, c)=\sum_{k=\alpha}^{n} \sum_{j=\alpha}^{k}\left(\begin{array}{l}
n \\
k
\end{array}\right)\left(\begin{array}{l}
k \\
j
\end{array}\right)(\ln c)^{n-k}\left(\ln \frac{b}{a}\right)^{j-\alpha}(-\alpha \ln a)^{k-j} x^{n-k} G_{j}^{(\alpha)} .
\end{gathered}
$$


Proof. By the Eq.(34) we can arrive at the Eq.(45) directly. It follows from Eq.(15) that

$$
\begin{aligned}
& G_{n}^{(\alpha)}(x ; a, b, c)=\frac{n !}{(n-\alpha) !} E\left[i\left(L^{(1)}+\cdots+L^{(\alpha)}\right) \ln \frac{b}{a}+x \ln c-\frac{\alpha}{2} \ln a b\right]^{n-\alpha} \\
& =\frac{n !}{(n-\alpha) !} \sum_{k=\alpha}^{n-\alpha}\left(\begin{array}{l}
n-\alpha \\
k-\alpha
\end{array}\right) E\left[i\left(L^{(1)}+\cdots+L^{(\alpha)}\right) \ln \frac{b}{a}-\frac{\alpha}{2} \ln a b\right]^{k-\alpha}(x \ln c)^{n-k} \\
& =\frac{n !}{(n-\alpha) !} \sum_{k=\alpha}^{n}\left(\begin{array}{l}
n-\alpha \\
k-\alpha
\end{array}\right)\left(\ln \frac{b}{a}\right)^{k-\alpha} E\left[i\left(L^{(1)}+\cdots+L^{(\alpha)}\right)-\frac{\alpha \ln a}{\ln b-\ln a}-\frac{\alpha}{2}\right]^{k-\alpha}(x \ln c)^{n-k} \\
& =\frac{n !}{(n-\alpha) !} \sum_{k=\alpha}^{n}\left(\begin{array}{l}
n-\alpha \\
k-\alpha
\end{array}\right) \frac{(k-\alpha) !}{k !} G_{k}^{(\alpha)}\left(\frac{\alpha \ln a}{\ln a-\ln b}\right) x^{n-k}(\ln c)^{n-k}\left(\ln \frac{b}{a}\right)^{k-\alpha} \\
& =\sum_{k=\alpha}^{n}\left(\begin{array}{l}
n \\
k
\end{array}\right)(\ln c)^{n-k}\left(\ln \frac{b}{a}\right)^{k-\alpha} x^{n-k} G_{k}^{(\alpha)}\left(\frac{\alpha \ln a}{\ln a-\ln b}\right),
\end{aligned}
$$

from which we see that

$$
\begin{aligned}
& E\left[i\left(L^{(1)}+\cdots+L^{(\alpha)}\right) \ln \frac{b}{a}-\frac{\alpha}{2} \ln a b\right]^{k-\alpha} \\
& =\left(\ln \frac{b}{a}\right)^{k-\alpha} E\left[i\left(L^{(1)}+\cdots+L^{(\alpha)}\right)-\frac{\alpha \ln a}{\ln b-\ln a}-\frac{\alpha}{2}\right]^{k-\alpha} \\
& =\left(\ln \frac{b}{a}\right)^{k-\alpha} \sum_{j=\alpha}^{k-\alpha}\left(\begin{array}{c}
k-\alpha \\
j-\alpha
\end{array}\right) E\left[i\left(L^{(1)}+\cdots+L^{(\alpha)}\right)-\frac{\alpha}{2}\right]^{j-\alpha}(-\alpha \ln a)^{k-j}\left(\ln \frac{b}{a}\right)^{j-k} \\
& =\sum_{j=\alpha}^{k}\left(\begin{array}{l}
k-\alpha \\
j-\alpha
\end{array}\right) \frac{(j-\alpha) !}{j !} G_{j}^{(\alpha)}\left(\ln \frac{b}{a}\right)^{j-\alpha}(-\alpha \ln a)^{k-j} .
\end{aligned}
$$

Hence we complete the proof of the theorem 11.

According to Eq.(45), Eq.(46) and Eq.(47) in theorem 11, we get the following theorem.

Theorem 12. Let $a, b$ and $c$ be positive integers with conditions $a \neq b, a b \neq 1$ and $c \neq 1$, for $\alpha \in \mathbb{N}_{+}, x \in \mathbb{R}, n \geq \alpha$, then we get

$$
\begin{gathered}
\sum_{k=\alpha}^{n}\left(\begin{array}{l}
n \\
k
\end{array}\right)(\ln c)^{n-k} x^{n-k} G_{k}^{(\alpha)}(a, b)=\sum_{k=\alpha}^{n}\left(\begin{array}{l}
n \\
k
\end{array}\right)(\ln c)^{n-k}\left(\ln \frac{b}{a}\right)^{k-\alpha} x^{n-k} G_{k}^{(\alpha)}\left(\frac{\alpha \ln a}{\ln a-\ln b}\right), \\
\sum_{k=\alpha}^{n}\left(\begin{array}{l}
n \\
k
\end{array}\right)(\ln c)^{n-k} x^{n-k} G_{k}^{(\alpha)}(a, b)=\sum_{k=\alpha}^{n} \sum_{j=\alpha}^{k}\left(\begin{array}{l}
n \\
k
\end{array}\right)\left(\begin{array}{l}
k \\
j
\end{array}\right) \frac{(\ln c)^{n-k}\left(\ln \frac{b}{a}\right)^{j-\alpha}}{(-\alpha \ln a)^{j-k}} x^{n-k} G_{j}^{(\alpha)} .
\end{gathered}
$$

We now turn to the above identities of the generalized higher-order Genocchi numbers and $x$. For the case $x$ is a constant variable, it seems normal. It is worth noticing 
that we can see $x$ as a random variable which obeys to a certain distribution and then take mathematical expectation on the two sides of the equation of random variables. It turns out that some interesting identities including the generalized higher-order Genocchi numbers and some combinatorial sequences are derived. The probabilistic method is much simpler and more effective here.

(1)Suppose that r.v $x=1-u_{1} u_{2}$, r.v $u_{1}, u_{2}, i . i . d \sim U[0,1]$, for the moment representation of the harmonic numbers $H_{n}$ in Eq.(7), the following identities hold true:

$$
\begin{aligned}
& \sum_{k=\alpha}^{n}\left(\begin{array}{l}
n \\
k
\end{array}\right)(\ln c)^{n-k} \frac{H_{n-k+1} G_{k}^{(\alpha)}(a, b)}{n-k+1}=\sum_{k=\alpha}^{n}\left(\begin{array}{l}
n \\
k
\end{array}\right)(\ln c)^{n-k}\left(\ln \frac{b}{a}\right)^{k-\alpha} \frac{H_{n-k+1} G_{k}^{(\alpha)}\left(\frac{\alpha \ln a}{\ln a-\ln b}\right)}{n-k+1}, \\
& \sum_{k=\alpha}^{n}\left(\begin{array}{l}
n \\
k
\end{array}\right)(\ln c)^{n-k} \frac{H_{n-k+1} G_{k}^{(\alpha)}(a, b)}{n-k+1}=\sum_{k=\alpha}^{n} \sum_{j=\alpha}^{k}\left(\begin{array}{l}
n \\
k
\end{array}\right)\left(\begin{array}{l}
k \\
j
\end{array}\right) \frac{(\ln c)^{n-k}\left(\ln \frac{b}{a}\right)^{j-\alpha}}{(-\alpha \ln a)^{j-k}} \frac{H_{n-k+1} G_{j}^{(\alpha)}}{n-k+1} .
\end{aligned}
$$

(2)Suppose that r.v $x=\Gamma-1, \Gamma \sim \Gamma(1,1)$, for the moment representation of the derangement numbers $d_{n}$ in Eq.(8), the following identities hold true:

$$
\begin{gathered}
\sum_{k=\alpha}^{n}\left(\begin{array}{l}
n \\
k
\end{array}\right)(\operatorname{lnc})^{n-k} d_{n-k} G_{k}^{(\alpha)}(a, b)=\sum_{k=\alpha}^{n}\left(\begin{array}{l}
n \\
k
\end{array}\right)(\ln c)^{n-k}\left(\ln \frac{b}{a}\right)^{k-\alpha} d_{n-k} G_{k}^{(\alpha)}\left(\frac{\alpha \ln a}{\ln a-\ln b}\right), \\
\sum_{k=\alpha}^{n}\left(\begin{array}{l}
n \\
k
\end{array}\right)(\ln c)^{n-k} d_{n-k} G_{k}^{(\alpha)}(a, b)=\sum_{k=\alpha}^{n} \sum_{j=\alpha}^{k}\left(\begin{array}{l}
n \\
k
\end{array}\right)\left(\begin{array}{l}
k \\
j
\end{array}\right) \frac{(\ln c)^{n-k}\left(\ln \frac{b}{a}\right)^{j-\alpha}}{(-\alpha \ln a)^{j-k}} d_{n-k} G_{j}^{(\alpha)} .
\end{gathered}
$$

(3)Suppose that r.v $x=\sqrt{5} u+\frac{(1-\sqrt{5})}{2}$, r.v $u \sim U[0,1]$, for the moment representation of the Fibonacci numbers $F_{n}$ in Eq.(9), the following identities hold true:

$$
\begin{aligned}
& \sum_{k=\alpha}^{n}\left(\begin{array}{l}
n \\
k
\end{array}\right)(\ln c)^{n-k} \frac{F_{n-k} G_{k}^{(\alpha)}(a, b)}{n-k+1}=\sum_{k=\alpha}^{n}\left(\begin{array}{l}
n \\
k
\end{array}\right)(\ln c)^{n-k}\left(\ln \frac{b}{a}\right)^{k-\alpha} \frac{F_{n-k} G_{k}^{(\alpha)}\left(\frac{\alpha \ln a}{\ln a-\ln b}\right)}{n-k+1}, \\
& \sum_{k=\alpha}^{n}\left(\begin{array}{l}
n \\
k
\end{array}\right)(\ln c)^{n-k} \frac{F_{n-k} G_{k}^{(\alpha)}(a, b)}{n-k+1}=\sum_{k=\alpha}^{n} \sum_{j=\alpha}^{k}\left(\begin{array}{l}
n \\
k
\end{array}\right)\left(\begin{array}{l}
k \\
j
\end{array}\right) \frac{(\ln c)^{n-k}\left(\ln \frac{b}{a}\right)^{j-\alpha}}{(-\alpha \ln a)^{j-k}} \frac{F_{n-k} G_{j}^{(\alpha)}}{n-k+1} .
\end{aligned}
$$

(4)Suppose that r.v $x=X$, r.v $X \sim P(1)$, for the moment representation of the Bell numbers $b_{n}$ in Eq.(10), the following identities hold true:

$$
\sum_{k=\alpha}^{n}\left(\begin{array}{l}
n \\
k
\end{array}\right)(\operatorname{lnc})^{n-k} b_{n-k} G_{k}^{(\alpha)}(a, b)=\sum_{k=\alpha}^{n}\left(\begin{array}{l}
n \\
k
\end{array}\right)(\operatorname{lnc})^{n-k}\left(\ln \frac{b}{a}\right)^{k-\alpha} b_{n-k} G_{k}^{(\alpha)}\left(\frac{\alpha \ln a}{\ln a-\ln b}\right),
$$




$$
\sum_{k=\alpha}^{n}\left(\begin{array}{l}
n \\
k
\end{array}\right)(\ln c)^{n-k} b_{n-k} G_{k}^{(\alpha)}(a, b)=\sum_{k=\alpha}^{n} \sum_{j=\alpha}^{k}\left(\begin{array}{l}
n \\
k
\end{array}\right)\left(\begin{array}{l}
k \\
j
\end{array}\right) \frac{(\ln c)^{n-k}\left(\ln \frac{b}{a}\right)^{j-\alpha}}{(-\alpha \ln a)^{j-k}} b_{n-k} G_{j}^{(\alpha)} .
$$

(5)Suppose that r.v $x=i L_{e}-\frac{1}{2}$, r.v $L_{1}, L_{2}, \cdots, i . i . d \sim L[0,1]$, let $L_{e}=\sum_{k \geq 1} \frac{L_{k}}{2 k \pi}$ be a random variable, for the moment representation of the Bernoulli numbers $B_{n}$ in Eq.(11), the following identities hold true:

$$
\begin{gathered}
\sum_{k=\alpha}^{n}\left(\begin{array}{l}
n \\
k
\end{array}\right)(\ln c)^{n-k} B_{n-k} G_{k}^{(\alpha)}(a, b)=\sum_{k=\alpha}^{n}\left(\begin{array}{l}
n \\
k
\end{array}\right)(\ln c)^{n-k}\left(\ln \frac{b}{a}\right)^{k-\alpha} B_{n-k} G_{k}^{(\alpha)}\left(\frac{\alpha \ln a}{\ln a-\ln b}\right), \\
\sum_{k=\alpha}^{n}\left(\begin{array}{l}
n \\
k
\end{array}\right)(\ln c)^{n-k} B_{n-k} G_{k}^{(\alpha)}(a, b)=\sum_{k=\alpha}^{n} \sum_{j=\alpha}^{k}\left(\begin{array}{l}
n \\
k
\end{array}\right)\left(\begin{array}{l}
k \\
j
\end{array}\right) \frac{(\ln c)^{n-k}\left(\ln \frac{b}{a}\right)^{j-\alpha}}{(-\alpha \ln a)^{j-k}} B_{n-k} G_{j}^{(\alpha)} .
\end{gathered}
$$

(6)Suppose that r.v $x=i L, L_{1}, L_{2}, \cdots, i . i . d \sim L[0,1]$, let r.v $L=\sum_{k \geq 1} \frac{L_{k}}{(2 k-1) \pi}$ be a random variable, for the moment representation of the Euler numbers $E_{n}$ in Eq.(12), the following identities hold true:

$$
\begin{aligned}
& \sum_{k=\alpha}^{n}\left(\begin{array}{l}
n \\
k
\end{array}\right)(\operatorname{lnc})^{n-k} \frac{E_{n-k} G_{k}^{(\alpha)}(a, b)}{2^{n-k}}=\sum_{k=\alpha}^{n}\left(\begin{array}{l}
n \\
k
\end{array}\right)(\ln c)^{n-k}\left(\ln \frac{b}{a}\right)^{k-\alpha} \frac{E_{n-k} G_{k}^{(\alpha)}\left(\frac{\alpha \ln a}{\ln a-\ln b}\right)}{2^{n-k}}, \\
& \sum_{k=\alpha}^{n}\left(\begin{array}{l}
n \\
k
\end{array}\right)(\ln c)^{n-k} \frac{E_{n-k} G_{k}^{(\alpha)}(a, b)}{2^{n-k}}=\sum_{k=\alpha}^{n} \sum_{j=\alpha}^{k}\left(\begin{array}{l}
n \\
k
\end{array}\right)\left(\begin{array}{l}
k \\
j
\end{array}\right) \frac{(\ln c)^{n-k}\left(\ln \frac{b}{a}\right)^{j-\alpha}}{(-\alpha \ln a)^{j-k}} \frac{E_{n-k} G_{j}^{(\alpha)}}{2^{n-k}} .
\end{aligned}
$$

(7)Suppose that r.v $x=X$, r.v $X \sim \Gamma(u, 1), u \sim U[0,1], X$ and $u$ are independent, for the moment representation of the Cauchy numbers $c_{n}$ in Eq.(13), the following identities hold true:

$$
\begin{gathered}
\sum_{k=\alpha}^{n}\left(\begin{array}{l}
n \\
k
\end{array}\right)(\operatorname{lnc})^{n-k} c_{n-k} G_{k}^{(\alpha)}(a, b)=\sum_{k=\alpha}^{n}\left(\begin{array}{l}
n \\
k
\end{array}\right)(\ln c)^{n-k}\left(\ln \frac{b}{a}\right)^{k-\alpha} c_{n-k} G_{k}^{(\alpha)}\left(\frac{\alpha \ln a}{\ln a-\ln b}\right), \\
\sum_{k=\alpha}^{n}\left(\begin{array}{l}
n \\
k
\end{array}\right)(\ln c)^{n-k} c_{n-k} G_{k}^{(\alpha)}(a, b)=\sum_{k=\alpha}^{n} \sum_{j=\alpha}^{k}\left(\begin{array}{l}
n \\
k
\end{array}\right)\left(\begin{array}{l}
k \\
j
\end{array}\right) \frac{(\ln c)^{n-k}\left(\ln \frac{b}{a}\right)^{j-\alpha}}{(-\alpha \ln a)^{j-k}} c_{n-k} G_{j}^{(\alpha)} .
\end{gathered}
$$

(8)Suppose that r.v $x=u_{1}+u_{2}+\cdots+u_{k}$, r.v $u_{1}, u_{2}, \cdots, i . i . d \sim U[0,1]$, for all $i$ and $n, k \geqslant 1$, for the moment representation of the Stirling numbers of second kind $S(n, k)$ in Eq.(14), the following identities hold true:

$$
\begin{gathered}
\sum_{k=\alpha}^{n}(\ln c)^{n-k} S(n, k) G_{k}^{(\alpha)}(a, b)=\sum_{k=\alpha}^{n}(\ln c)^{n-k}\left(\ln \frac{b}{a}\right)^{k-\alpha} S(n, k) G_{k}^{(\alpha)}\left(\frac{\alpha \ln a}{\ln a-\ln b}\right), \\
\sum_{k=\alpha}^{n}(\ln c)^{n-k} S(n, k) G_{k}^{(\alpha)}(a, b)=\sum_{k=\alpha}^{n} \sum_{j=\alpha}^{k}\left(\begin{array}{c}
k \\
j
\end{array}\right) \frac{(\ln c)^{n-k}\left(\ln \frac{b}{a}\right)^{j-\alpha}}{(-\alpha \ln a)^{j-k}} S(n, k) G_{j}^{(\alpha)} .
\end{gathered}
$$




\section{Acknowledgements}

The research is supported by the Natural Science Foundation of China under Grant 11461050 and Natural Science Foundation of Inner Mongolia under Grant 2016MS0104.

\section{References}

[1] S. Araci, W.A. Khan, and M. Acikgoz et al. A New Generalization of Apostol Type Hermite-Genocchi Polynomials and Its Applications. Springerplus, 5(1):1-17, 2016.

[2] S. Gaboury and B. Kurt. Some Relations Involving Hermite-Based Apostol-Genocchi Polynomials. Applied Mathematics Sciences, 6(82):4091-4102, 2012.

[3] Y. He, S. Araci, H.M. Srivastava, and M. Acikgoz. Some New Identities for the Apostol-Bernoulli Polynomials and the Apostol-Genocchi Polynomials. Applied Mathematics and Computation, 262(C):31-41, 2015.

[4] H. Jolany, R.E. Alikelaye, and S.S. Mohamad. Some Results on the Generalization of Bernoulli, Euler and Genocchi Polynomials. Acta Univ.apulensis Math.inform, 27(27):299-306, 2011.

[5] H. Jolany, H. Sharifi, and R.E. Alikelaye. Some Results for the Apostol-Genocchi Polynomials of Higher Order. Bulletin of the Malaysian Mathematical Society, 36(36):465479, 2011.

[6] W.A. Khan. A New Class of Hermite Poly-Genocchi Polynomials. Journal of Analysis and Number Theory, 4:1-8, 2016.

[7] W.A. Khan, S. Araci, M. Acikgoz, and H. Haroon. A New Class of Partially Degenerate Hermite-Genocchi Polynomials. Journal of Nonlinear Sciences and Applications, 10(9):5072-5081, 2017.

[8] W.A. Khan and M. Ghayasuddin. Some Symmetric Identities for the Generalized Hermite-Euler and Hermite-Genocchi Polynomials. Journal of Analysis and Number Theory, 5(2):1-7, 2017.

[9] W.A. Khan and H. Haroon. Some Symmetric Identities for the Generalized Bernoulli, Euler and Genocchi Polynomials Associated with Hermite Polynomials. Springer Plus, 5(1):1-21, 2016.

[10] Q.M. Luo, F. Qi, and L. Debnath. Generalizations of Euler numbers and polynomials. International Journal of Mathematics and Mathematical Sciences, 2003(61):38933901, 2003.

[11] M.A. Pathan and W.A. Khan. A New Class of Generalized Polynomials Associated with Hermite and Euler Polynomials. Mediterranean Journal of Mathematics, 13(3):913-928, 2016. 
[12] H.M. Srivastava. Some Generalizations and Basic (or q-) Extensions of the Bernoulli, Euler and Genocchi Polynomials. Applied Mathematics and Information Sciences, $5(3): 390-444,2010$.

[13] P. Sun. Moment Representation of Bernoulli Polynomial, Euler Polynomial and Gegenbauer Polynomials. Statistics and Probability Letters, 77:748-751, 2007.

[14] P. Sun and T. Wang. Probabilistic Representation with Application of Stirling Numbers. Acta Mathematica Sinica Chinese Series, 41(2):281-290, 1998.

[15] H.S. Wilf. Generating Functionology. Academic Press, New York, 1990. 